BNL-NT-01/12

NT@UW-01-010

TAUP-2682-2001

\title{
Instantons in the Saturation Environment
}

\author{
Dmitri Kharzeev, ${ }^{1}$ Yuri V. Kovchegov, ${ }^{2}$ Eugene Levin ${ }^{3}$ \\ ${ }^{1}$ Physics Department, Brookhaven National Laboratory \\ Upton, NY 11973, USA \\ 2 Department of Physics, University of Washington, Box 351560 \\ Seattle, WA 98195, USA \\ ${ }^{3}$ HEP Department, School of Physics and Astronomy \\ Tel Aviv University, Tel Aviv 69978, Israel
}

\begin{abstract}
We show that instanton calculations in QCD become theoretically well defined in the gluon saturation environment which suppresses large size instantons. The effective cutoff scale is determined by the inverse of the saturation scale. We concentrate on two most important cases: the small- $x$ tail of a gluon distribution of a high energy hadron or a large nucleus and the central rapidity region in a high energy hadronic or heavy ion collision. In the saturation regime the gluon density in a single large ultrarelativistic nucleus is high and gluonic fields are given by the classical solutions of the equations of motion. We show that these strong classical fields do not affect the density of instantons in the nuclear wave function compared to the instanton density in the vacuum. A classical solution with nontrivial topological charge is found for the gluon field of a single nucleus at the lowest order in the instanton perturbation theory. In the case of ultrarelativistic heavy ion collisions a strong classical gluonic field is produced in the central rapidity region. We demonstrate that this field introduces a suppression factor of $\exp \left\{-c \rho^{4} Q_{s}^{4} /\left[8 \alpha_{s}^{2} N_{c}\left(Q_{s} \tau\right)^{2}\right]\right\}$ in the instanton size distribution, where $Q_{s}$ is the saturation scale of both (identical) nuclei, $\tau$ is the proper time and $c \approx 1$ is the gluon liberation coefficient. This factor suggests that gluonic saturation effects at the early stages of nuclear collisions regulate the instanton size distribution in the infrared region and make the instanton density finite by suppressing large size instantons.
\end{abstract}

\section{INTRODUCTION}

At very high energies corresponding to extremely small values of Bjorken $x$ variable the density of partons in the hadronic or nuclear light cone wave function can get very large leading to the effect of saturation of gluon and quark distributions [1]. The large density of partons in the transverse plane produces strong gluonic fields $A_{\mu} \sim 1 / g$ which leads to a number of interesting non-linear phenomena. The transition to the saturation regime in the $\left(x, Q^{2}\right)$ plane is characterized by the saturation scale $Q_{s}(x)$. It has been shown in [2] that for a large nucleus the saturation scale grows with the nuclear atomic number as $Q_{s}^{2} \sim A^{1 / 3}$, and thus for a heavy ion it can get large $\left(Q_{s}^{2} \gg \Lambda_{Q C D}^{2}\right)$ making the strong coupling constant small $\alpha_{s}\left(Q_{s}\right) \ll 1$. This allowed McLerran and Venugopalan [4] to argue that gluonic fields in the saturation region are given by the solution of the classical Yang-Mills equations of motion in the presence of the source given by the ultrarelativistic nucleus on the light cone. The solution of the classical Yang-Mills equations for a large nucleus (non-Abelian Weizsäcker-Williams field) has been found in [6,77]. It has been shown [2, [7.8] that 
saturation regularizes the power-law divergence of the classical unintegrated gluon distribution function in the infrared region. The unintegrated gluon distribution at the two-gluon level is proportional to $1 / k_{\perp}^{2}$ and diverges for small $k_{\perp}$. The classical field resums all multiple rescattering effects, which corresponds to summing up powers of $Q_{s} / k_{\perp}$. The resulting unintegrated gluon distribution given by the non-Abelian Weizsäcker-Williams field of a large nucleus is proportional to $\ln Q_{s} / k_{\perp}$ in the small transverse momentum region [7,8]. Thus the infrared singularity becomes only logarithmic and integrable. As we go towards smaller values of $x$ quantum corrections to the classical field become important. The summation of leading logarithmic $(\ln 1 / x)$ corrections to the classical multiple rescattering picture led to a non-linear evolution equation for the total scattering cross section of a color dipole on a hadron or nucleus which was derived independently by one of the authors in [9] using Mueller's dipole model of [10] and by Balitsky in [11] employing the high energy effective lagrangian technique. Similar equation has emerged recently out of renormalization group approach [12,13]. The solution of this non-linear evolution equation should give the behavior of the hadronic cross sections at very high energies and specify the dependence of $Q_{s}$ on $x$. The solution has been found by approximate analytical methods [9, 14 and by numerical simulations [15, 16] yielding us with the saturation scale which is a growing function of energy, $Q_{s} \sim 1 / x^{\delta}$, where $\delta$ is close to the value of the BFKL pomeron intercept [17. Thus at very high energies the saturation scale can get large even for a hadron making the small- $x$ tail of the gluon distribution in hadrons similar to the small- $x$ tail of the gluon distribution in large nuclei.

With a large fraction of high energy scattering data being attributed to some non-perturbative QCD phenomena, instantons [18 21] might play an important role in high energy scattering processes [22]. There have been developed several techniques of calculating the instanton-mediated scattering amplitudes including the effective instanton lagrangian approach [23,24] and the valley method [25]. Instanton-induced effects could be important to deep inelastic scattering processes and other high energy processes as was shown in [26 28]. Several years ago there has been developed a vigorous activity studying the possibility of baryon number violating effects due to instantons in electroweak theory [29]. Recently the authors suggested that instanton-induced interactions could play a key role in the dynamics of soft pomerons in hadronic scattering cross sections and in multi-particle production [31] (see also [32]).

The role of instantons in heavy ion collisions has not been studied in such detail. It has been recently suggested [33] that the instanton-induced particle production can account for a significant fraction of the total charged particle multiplicity in the heavy ion collisions at RHIC [34]. Since it has been argued that the small- $x$ gluons are most important for the mid-rapidity particle production in heavy ion collisions [35] 38] it is natural to investigate whether instanton-induced effects are important for these small- $x$ gluons, which is our main goal here.

The qualitative picture of what one might expect to happen to instantons in the saturation environment is as follows. Let us consider a gluon propagating through the strong background gluonic field created either by a single large nucleus or in a collision of two nuclei. The gluon would undergo multiple rescatterings in the field which would have the physical effect of generating some non-zero effective mass for the gluon, which would be roughly proportional to $Q_{s}$ (see, for instance, [39]). Equivalently we can say that due to multiple rescatterings the gluons are screened on the transverse distances inversely proportional to the effective mass, i.e., on the distances of the order of $1 / Q_{s}$. Here we can draw an analogy to the case of QCD at high finite temperature $T$. There the Debye mass of the gluons in quark-gluon plasma $m_{D} \sim g T$ introduces screening on the distances of the order of $1 / m_{D} \sim 1 / g T$ which in turn suppresses large size instantons by the factor of 40]

$$
e^{- \text {const } \rho^{2} m_{D}^{2}} \sim e^{- \text {const } \rho^{2} \rho^{2}} .
$$

In these approximate estimates we are not keeping track of the factors of $g, N_{C}$ and $N_{f}$, putting them to be some constants. Thus arguing that the saturation scale plays the role of Debye screening mass for small- $x$ gluons $m_{D} \sim Q_{s}$ [41] we might expect that the instanton size distribution in the saturation background would also obtain a suppression factor of

$$
e^{-\operatorname{const} \rho^{2} Q_{s}^{2}}
$$

for large instantons. This would imply that instantons with sizes $\rho \gtrsim \rho_{0} \sim 1 / Q_{s}$ are exponentially suppressed and only small instantons with sizes $\rho \lesssim \rho_{0}$ can contribute to the scattering processes. This would justify 
the perturbative calculation we are about to perform below, since the strong coupling constant for small size instantons is small $\alpha_{s}\left(\rho<1 / Q_{s}\right) \ll 1$.

Suppression of large size instantons could be anticipated based on the similar arguments related to the philosophy introduced in [4]: the typical size of the nuclear color charge fluctuations in the transverse plane is of the order of $1 / Q_{s}$. Thus instantons of larger size would just be washed out by the color charge fluctuations in the ultrarelativistic nucleus.

An analogy can also be drawn with QCD at finite density and zero temperature. There the instantons are again suppressed by an exponential factor of 42.

$$
e^{- \text {const } \rho^{2} \mu^{2}}
$$

The suppression is due to the following qualitative picture: large size instantons tend to produce quarks with momenta $k \sim 1 / \rho \lesssim \mu$ and this process is suppressed at large $\mu$ due to Pauli blocking since all the quark energy levels with momenta $k \leq \mu$ are occupied 1 . Instantons in the saturation environment produce gluons, which are of course bosons and do not have any Pauli blocking effects. The role of Pauli blocking for gluons is played by the distribution of gluons in the saturation wave function of a single nucleus which is very similar (at the qualitative level) to the Fermi-Dirac distribution. In the case of nuclear collision the transverse momentum spectrum of produced gluons also exhibits saturation behavior and levels off in the infrared resembling Fermi-Dirac distribution [38]. Due to multiple rescatterings the gluons with lower transverse momenta get pushed towards higher transverse momenta of the order of $k_{\perp} \sim Q_{s}$. Thus there are very few gluons left with small $k_{\perp}$ and the large size instanton-like solutions which would tend to produce those should be suppressed. Associating the width of the nuclear gluon distribution with the chemical potential of the Fermi-Dirac distribution $Q_{s} \sim \mu$ we would again arrive at Eq. (2).

The intuitive arguments presented above suggest that the interaction with gluons in the saturation environment will exponentially suppress the instantons with large size (larger than $1 / Q_{s}(x)$ ). Such suppression would result in an infrared safe and, therefore, well defined theoretically instanton calculus in the saturation medium. To show that this is indeed the case is the main objective of this paper.

The paper is organized as follows. In Sect. II we present the general techniques that we are going to use. We will briefly review the solution of the problem of small instantons in a slowly varying background field using the effective instanton lagrangian approach [23,24]. We show that the background field affects the instanton size distribution only if it has a non-zero value of $G_{\mu \nu}^{a 2}$ (or $G_{\mu \nu}^{a} \tilde{G}_{\mu \nu}^{a}$ ) at the space-time point of interest [23:24].

We will proceed in Sect. III by addressing the issue of instanton size distribution in the small- $x$ tail of the gluon distribution in a single nucleus or hadron. Throughout most of this paper we will consider the classical picture of saturation. We are interested in the small- $x$ tail of the distribution with $x$ small enough for multiple rescattering effects to be become important [4, 6, 8 . For this we require the coherence length of a small- $x$ gluon to be comparable to nuclear size $l_{c} \approx 1 / 2 m x \gtrsim 2 R$, which yields us with $x \lesssim 1 / 4 m R$ [2, 43, 44]. At the same time the value of $x$ should not be too small since we do not want the quantum corrections to start playing an important role. Thus we want $\alpha_{s} \ln 1 / x \lesssim 1$ which leads to $x \gtrsim \exp \left(-1 / \alpha_{s}\right)$. In Sect. IIIA we demonstrate that the classical gluon field of a single hadron or nucleus has zero value of $G_{\mu \nu}^{a 2}$ and does not affect the instanton size distribution, introducing no enhancement or suppression of instanton effects. We proceed in Sect. IIIB by calculating the one loop leading logarithmic $(\ln 1 / x)$ correction to the classical field of the nucleus. For this correction to become important we have to relax the $\alpha_{s} \ln 1 / x \lesssim 1$ condition. We observe that even after inclusion of this correction the field strength squared $G_{\mu \nu}^{a 2}$ of the gluon field still remains zero and does not affect instanton distribution. We relate this observation to the representation of quantum evolution as a series of classical emissions as advocated in [10,12 and based on that argue that our result is true to all orders in evolution resumming powers of $\alpha_{s} \ln 1 / x[9]$ [1].

The classical field of a large nucleus found in [6.] was a non-topological solution, in the sense that it did not create a transition from a region of one topological charge to a region of a different topological charge. In this paper we would like to address the issue whether there exists a topological solution of the classical YangMills equations of motion in the presence of external source given by the large nucleus. In Sect. III we show

\footnotetext{
${ }^{1} \mathrm{Yu}$. K. would like to thank Larry Yaffe for providing this argument.
} 
that this solution, if exists, is just as probable as the usual "non-topological" solution of [6,7]. Unfortunately we were unable to construct an exact instanton-like solution for this problem. Instead in Sect. IIIB we are going to consider a QCD instanton perturbatively interacting with the classical field of the nucleus [6,7] and producing a combined classical field of the instanton-nucleus configuration which would now connect regions of different topological charge. This field squared contributes to the one loop correction to $G_{\mu \nu}^{a 2}$, and, if viewed as a field of the nucleus probed by a point-like instanton is not a classical field anymore. However the field itself is a classical field of the instanton-nucleus configuration. This classicality is due to the fact that we will be performing the calculation resumming all powers of the parameter $\alpha_{s}^{2} A^{1 / 3}$ (or, equivalently, $Q_{s}^{2} / k_{\perp}^{2}$ ) and neglecting higher order corrections in $\alpha_{s}$ to it 6 , 8 . The equivalence between the classical field techniques and this resummation has been discussed in 4.6.68,35 38 and references therein.

In Sect. IV we will consider the case of hadronic or nuclear collisions. As has been argued in [35] the dominant particle production mechanism for central rapidity gluons could be due to the strong classical gluon field produced by the colliding hadrons or nuclei. The field is again characterized by the saturation scale of the colliding nuclei $Q_{s}$. This gluonic field has been calculated at the lowest order in perturbation theory in [35,36] and analyzed numerically in [37. Recent progress in calculating the produced particle spectrum due to the classical field was made in [38]. The classical field produced by colliding nuclei has a non-vanishing value of $G_{\mu \nu}^{a 2}$ and therefore influences the instanton size distribution. By a direct calculation using the lowest order classical field [35.,36] we demonstrate in Sect. IV that the effect of this classical fields would be to suppress large size instantons by a suppression factor of

$$
\exp \left(-\frac{c \rho^{4} Q_{s}^{4}}{8 \alpha_{s}^{2} N_{c}\left(Q_{s} \tau_{0}\right)^{2}}\right)
$$

with the proper time of the instanton position $\tau_{0}=\sqrt{2 x_{0+} x_{0-}}$ and $c$ the gluon liberation coefficient. $x_{0}$ is the space-time point where we measure the instanton density. Eq. (位) shows that at large proper time $(\tau \rightarrow \infty)$, long after the collision, the lagrangian density $G_{\mu \nu}^{a 2}$ at each particular point gets small and large instantons will not be suppressed anymore. The amount of suppression depends on the gluon liberation coefficient, which was estimated numerically to be $c=1.29 \pm 0.09$ [37] and analytically to be $c \approx 2 \ln 2$ [38], while RHIC data [34] suggests $c=1.23 \pm 0.20$ [45]. We will also propose that the instanton suppression of Eq. (4) at extremely high energies might lead to suppression and, therefore, unitarization of the soft pomeron of [31. We will conclude the paper by estimating that the saturation effects reduce the instanton density in the central rapidity region at RHIC by three orders of magnitude compared to the instanton density in vacuum as extracted from lattice data [27].

\section{INSTANTONS IN BACKGROUND FIELD}

The problem of small instantons in a slowly varying background field was first addressed in [23,24] and was

resolved by introducing the effective instanton lagrangian $L_{\text {eff }}^{I(\bar{I})}(x)$. The complete field of a single instanton solution could be reconstructed by perturbatively resumming the powers of the effective instanton lagrangian which corresponds to perturbation theory in powers of the instanton size parameter $\rho^{2}$. In our case here the background field arises due to the strong source current $J_{\mu}^{a}$. The current will be due to a single nucleus (Sect. III) and two colliding nuclei (Sect. IV). Perturbative resummation of powers of the source current term translates itself into resummation of the powers of the classical field parameter $\alpha_{s}^{2} A^{1 / 3}$ [4, 6 . Thus the problem of instantons in the background classical gluon field is described by the effective action in Minkowski space

$$
S_{e f f}=\int d^{4} x\left(-\frac{1}{4} G_{\mu \nu}^{a}(x) G_{\mu \nu}^{a}(x)+L_{e f f}^{I}(x)+L_{e f f}^{\bar{I}}(x)+J_{\mu}^{a} A_{\mu}^{a}(x)\right) .
$$

In Eq. (5) the point-like instanton vertices are given by the instanton-induced effective lagrangian [23, 24, 48]

$$
L_{e f f}^{I}\left(x_{0}\right)=\int d \rho n_{0}(\rho) d R \exp \left(-\frac{2 \pi^{2}}{g} \rho^{2} \bar{\eta}_{a \mu \nu}^{M} R^{a a^{\prime}} G_{\mu \nu}^{a^{\prime}}\left(x_{0}\right)\right)
$$

in which $n_{0}(\rho)$ is the instanton size distribution function in vacuum given by 49.50 


$$
n_{0}(\rho)=\frac{0.466 e^{-1.679 N_{c}}}{\left(N_{c}-1\right) !\left(N_{c}-2\right) !} \frac{1}{\rho^{5}}\left(\frac{2 \pi}{\alpha_{s}(\rho)}\right)^{2 N_{c}} e^{-\frac{2 \pi}{\alpha_{s}(\rho)}}
$$

where $b=(11 / 3) N_{c}-(2 / 3) N_{f}$. In Eq. (6) $\bar{\eta}_{a \mu \nu}^{M}$ is the 't Hooft symbol in Minkowski space defined in terms of the usual 't Hooft symbol in euclidean space by [48]

$$
\bar{\eta}_{a \mu \nu}^{M}=\left\{\begin{array}{cc}
\bar{\eta}_{a \mu \nu}, & \mu, \nu=1,2,3 \\
i \bar{\eta}_{a 4 \nu}, & \mu=0, \nu=1,2,3 .
\end{array}\right.
$$

$x_{0}$ in Eq. (6) is the position of the instanton and $R^{a a^{\prime}}$ is the matrix of rotations in the color space with $d R$ denoting the averaging over instanton color orientations. To obtain the effective lagrangian for antiinstantons from Eq. (6) one has to change $\bar{\eta}_{a \mu \nu}^{M}$ into $\eta_{a \mu \nu}^{M}$ in it $\left(\eta_{a \mu \nu}^{M}=\left(\bar{\eta}_{a \mu \nu}^{M}\right)^{*}\right)$.

The classical current of a single large ultrarelativistic nucleus in the effective action of Eq. (5) is given in McLerran-Venugopalan model by [4,6, 6 ]

$$
J_{\mu}^{a}=\delta_{\mu+} \delta\left(x_{-}\right) \rho^{a}\left(x_{\perp}\right),
$$

where $\rho^{a}\left(x_{\perp}\right)$ is the two-dimensional color charge density of the nucleus. In the calculations of diagrams below we will be using the explicit model of the nucleus as consisting of independent nucleons which is justified at high energies and is equivalent to the description of the nucleus in terms of the current of Eq. (9) [6, 8]. In the case of two colliding nuclei the source current will be

$$
J_{\mu}^{a}=\delta_{\mu+} \delta\left(x_{-}\right) \rho_{1}^{a}\left(x_{\perp}\right)+\delta_{\mu-} \delta\left(x_{+}\right) \rho_{2}^{a}\left(x_{\perp}\right),
$$

with $\rho_{1}^{a}\left(x_{\perp}\right)$ and $\rho_{2}^{a}\left(x_{\perp}\right)$ the color charge densities of the colliding nuclei.

The action of Eq. (5) solves the problem of topologically non-trivial classical fields of nuclei at the conceptual level. It allows one to construct a perturbative series in the powers of $\rho^{2}$ and $\alpha_{s}^{2} A^{1 / 3}$ which, with proper regularization of singularities, should sum up to yield us the classical field of a nucleus or nuclei with non-zero topological charge. In Sect. IIIB we will construct an example of such field in the single instanton sector at the lowest order in these parameters.

Following [23.24] we may write the first correction in $\rho$ to the instanton size distribution generated by the background field of the nucleus or nuclei as

$$
\begin{gathered}
n_{\text {sat }}(\rho)=\left(A\left|L_{\text {eff }}^{I}\right| A\right)= \\
=n_{0}(\rho)\left(1+\frac{\pi^{3} \rho^{4}}{\alpha_{s}\left(N_{c}^{2}-1\right)}\left(A\left|G_{\mu \nu}^{a}\left(x_{0}\right) G_{\mu \nu}^{a}\left(x_{0}\right)-G_{\mu \nu}^{a}\left(x_{0}\right) \tilde{G}_{\mu \nu}^{a}\left(x_{0}\right)\right| A\right)+\text { higher orders in } \rho^{4}\right)
\end{gathered}
$$

where $G_{\mu \nu}^{a}\left(x_{0}\right)$ is the field strength of the slowly varying background field taken at the position of the instanton and $\tilde{G}_{\mu \nu}^{a}=(1 / 2) \epsilon_{\mu \nu \rho \sigma} G_{\rho \sigma}^{a}$ is the dual field strength. $(A|\ldots| A)$ denotes averaging in the nuclear wave function(s) [4.6]. Eq. (11) could be obtained by expanding the effective lagrangian $L_{\text {eff }}^{I}$ in the powers of $\rho^{2}$ and averaging the resulting terms in the nuclear wave function and over instanton orientations [23, 24, 48]. As was shown in [6] for the classical field of a large nucleus at the leading powers in $A$ the higher order correlators factorize (Gaussian averaging) yielding the property which was assumed for the vacuum fields in [24]

$$
\begin{aligned}
& \left(A\left|\bar{\eta}_{a_{1} \mu_{1} \nu_{1}}^{M} R^{a_{1} a_{1}^{\prime}} G_{\mu_{1} \nu_{1}}^{a_{1}^{\prime}} \ldots \bar{\eta}_{a_{2 k} \mu_{2 k} \nu_{2 k}}^{M} R^{a_{2 k} a_{2 k}^{\prime}} G_{\mu_{2 k} \nu_{2 k}}^{a_{2 k}^{\prime}}\right| A\right)= \\
& =(2 k-1) ! !\left[\left(A\left|\bar{\eta}_{a \mu \nu}^{M} R^{a a^{\prime}} G_{\mu \nu}^{a^{\prime}} \bar{\eta}_{b \alpha \beta}^{M} R^{b b^{\prime}} G_{\alpha \beta}^{b^{\prime}}\right| A\right)\right]^{k} .
\end{aligned}
$$

Using the color neutrality of a nucleus we write 


$$
\left(A\left|G_{\mu \nu}^{a^{\prime}} G_{\alpha \beta}^{b^{\prime}}\right| A\right)=\frac{\delta^{a^{\prime} b^{\prime}}}{N_{c}^{2}-1}\left(A\left|G_{\mu \nu}^{a} G_{\alpha \beta}^{a}\right| A\right) .
$$

Employing Eq. (13) in Eq. (12) together with the orthogonality of the color rotation matrices $R^{a a^{\prime}} R^{b a^{\prime}}=\delta^{a b}$ and performing some simple algebra of 't Hooft symbols one can show that the effect of higher order terms in $\rho^{4}$ in Eq. (11) is just to exponentiate the lowest order term leading to (cf. [23,24)

$$
n_{s a t}(\rho)=n_{0}(\rho) \exp \left[\frac{\pi^{3} \rho^{4}}{\alpha_{s}\left(N_{c}^{2}-1\right)}\left(A\left|G_{\mu \nu}^{a}\left(x_{0}\right) G_{\mu \nu}^{a}\left(x_{0}\right)-G_{\mu \nu}^{a}\left(x_{0}\right) \tilde{G}_{\mu \nu}^{a}\left(x_{0}\right)\right| A\right)\right] .
$$

Thus in order to find the effect of a particular background field on the instanton size distribution all one has to do is to calculate the matrix element in the power of the exponent in Eq. (14). The effective instanton lagrangian approach is strictly valid only for instantons of the size much smaller than the typical variation of the external field [24] though it usually works for larger instantons too. In the case of saturation background there is only one dimensional parameter characterizing the external field and it is the saturation scale $Q_{s}$. Thus one might argue that the matrix element of $G_{\mu \nu}^{a 2}$ is proportional to $Q_{s}^{4}$ and therefore Eq. (14) resums all powers of the parameter $\rho^{4} Q_{s}^{4}$ and is strictly speaking valid only when

$$
\rho^{4} Q_{s}^{4} \ll 1 .
$$

This is the approximation that we are going to employ throughout the paper: the instantons we consider are much smaller than $1 / Q_{s}$. This assumption also keeps $\alpha_{s}(\rho)$ small allowing us to use perturbation theory.

Eq. (48) includes only the dipole interaction term between the instanton and the background field. Higher order multipole interactions in general should also be included. However, since these interactions would correspond to subleading in $A$ correction to factorization of Eq. (12) the multipole terms would also be subleading in $A$. They would have less powers of $A^{1 / 3}$ per power of $\rho^{4}$ than the leading (dipole) term and could be neglected.

\section{SINGLE NUCLEUS CASE}

\section{A. Lowest Order Diagrams}

Let us start constructing the modified instanton size distribution in the external field of a single nucleus along with the field of the instanton-nucleus configuration using the action of Eq. (5). We will consider a single instanton interacting with the classical field of a large ultrarelativistic nucleus. At the lowest orders in $\rho^{2}$ and $g$ the field of the I-nucleus configuration would be given simply by the sum of the fields of the instanton and the nucleus, similarly to the sum ansatz for the I-I configurations [51]. Since we are interested in the effect of the nuclear gluon field on the instanton size distribution it is more convenient to start analyzing diagrams for the interaction of the instanton with the field directly, without first deriving the field. This is equivalent to calculating the matrix element in the exponent of Eq. (14). We will also concentrate for now on the case of a single rescattering in the nucleus, which parametrically corresponds to the case of $\alpha_{s}^{2} A^{1 / 3} \lesssim 1$. We will generalize our results to the $\alpha_{s}^{2} A^{1 / 3} \sim 1$ case after we obtain the lowest order expression.

All the graphs that we are going to analyze will be calculated in $\partial_{\mu} A_{\mu}=0$ light cone gauge. The lowest order diagram which might contribute to the action of the I-nucleus configuration is the one gluon exchange diagram depicted in Fig. 1 A. There an instanton interacts with one nucleon in the nucleus which is moving ultrarelativistically with the large momentum in the light cone "+" direction. To obtain the average value of the action we have to average this diagram (as well as all others) over the nuclear wave function [4.66 8]. The procedure includes averaging over all possible positions of nucleons in nuclei and quarks in nucleons, as well as averaging in the color spaces of each particular nucleon, which makes sure that the nucleus is in the color singlet state both before and after the interaction [6]. Averaging in the color space on the nucleon in figure Fig. 11A gives zero for this diagram since $\operatorname{Tr} T^{a}=0$.

Similar color arguments can rule out a number of diagrams with extra gluons involved where only one gluon interacts with the nucleon. Diagram in Fig. $1 \mathrm{~B}$ has two gluons interacting with the nucleon and can not be proved to vanish by simple color algebra. Adding the diagram with the gluon lines crossed to it and performing color averaging one can see that the diagram is proportional to 


$$
\begin{gathered}
\delta\left(\left(p-l-l^{\prime}\right)^{2}\right) R^{a a^{\prime}} \bar{\eta}_{a^{\prime}-\mu}^{M} l_{\mu} R^{b b^{\prime}} \eta_{b^{\prime}-\nu}^{M} l_{\nu}^{\prime} \delta^{a b}\left(\tilde{u}\left(p-l-l^{\prime}\right) \gamma_{+} \frac{\gamma \cdot(p-l)}{(p-l)^{2}+i \epsilon} \gamma_{+} \tilde{u}(p)\right. \\
\left.+\tilde{u}\left(p-l-l^{\prime}\right) \gamma_{+} \frac{\gamma \cdot\left(p-l^{\prime}\right)}{\left(p-l^{\prime}\right)^{2}+i \epsilon} \gamma_{+} \tilde{u}(p)\right)
\end{gathered}
$$

where $p$ is the momentum of the quark line in the nucleon below having a large $p_{+}$component. All the other light cone components of the gluon's momenta in the diagrams we consider are much smaller than that $l_{+}, l_{+}^{\prime} \ll p_{+}[6]$. Using the orthogonality of the color rotation matrices $R^{a a^{\prime}} R^{a b^{\prime}}=\delta^{a^{\prime} b^{\prime}}$ and evaluating the products of $\gamma$-matrices in the eikonal approximation one can show that Eq. (16) is proportional to

$$
\bar{\eta}_{a-\mu}^{M} l_{\mu} \eta_{a-\nu}^{M} l_{\nu}^{\prime} \delta\left(l_{-}+l_{-}^{\prime}\right)\left(\frac{1}{l_{-}-i \epsilon}-\frac{1}{l_{-}+i \epsilon}\right) \sim l_{-} l_{-}^{\prime} \delta\left(l_{-}\right) \delta\left(l_{-}^{\prime}\right)=0 .
$$

Thus we have shown that the contribution of the diagram in Fig. 1 $1 \mathrm{~B}$ to the action of the I-Nucleus system is also zero.

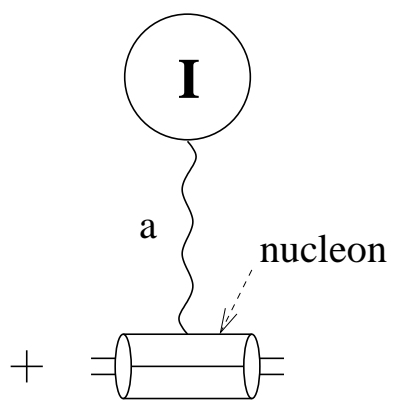

A

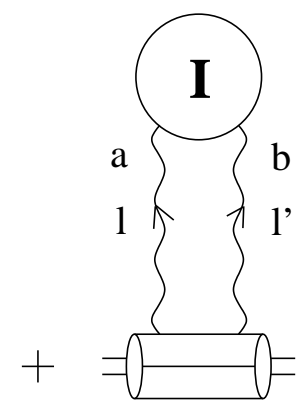

B

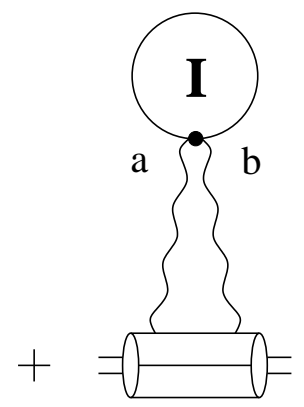

$\mathbf{C}$

FIG. 1. Lowest order diagrams which might contribute to the instanton action in the field of a nucleus. Only one nucleon participates in the interaction here. After a simple calculation (see text) one can see that the contributions of these diagrams are zero.

We have to point out that due to the non-Abelian structure of the effective instanton lagrangian of Eq. (6) there is another type of an instanton vertex with two gluons fusing into an instanton at the same point in coordinate space [30], which yields us with another diagram which might contribute to the instantonnucleus interactions as shown in Fig. 1]C. However the vertex is antisymmetric in the color indices of the gluons connecting to it. Due to color averaging in the nucleon below the diagram in Fig. 1] $\mathrm{C}$ is proportional to $f^{a b c} \delta^{a b}=0$ and does not contribute to the action.

The reason why all the diagrams in Fig. 1 are zero could be understood in terms of the effective instanton lagrangian result given by Eq. (14). At the leading (classical) order the value of the matrix elements of the local operators $G_{\mu \nu}^{a}\left(x_{0}\right) G_{\mu \nu}^{a}\left(x_{0}\right)$ and $G_{\mu \nu}^{a}\left(x_{0}\right) \tilde{G}_{\mu \nu}^{a}\left(x_{0}\right)$ in the nuclear wave function could be obtained by just substituting the field strength of the classical non-Abelian Weizsäcker-Williams field of a nucleus in it. As could be seen from the exact expression for this field given in [6] the only non-vanishing components of its field strength tensor are $G_{+\perp}^{W W a}$, so that $\left(G_{\mu \nu}^{W W a}\right)^{2}=G_{\mu \nu}^{W W a} \tilde{G}_{\mu \nu}^{W W a}=0$. Therefore from Eq. (14) it follows that there is no correction to the instanton density if one calculates the matrix elements of $G^{2}$ and $G \tilde{G}$ only at the classical level. However, there exists a possibility that higher order in $\alpha_{s}$ corrections might yield a non-zero value for these matrix elements. To check this we will now go one step beyond and calculate what appears like a one loop correction to this classical result.

\section{B. Next-to-Leading Order Diagrams}

In this subsection we are going to analyze two-loop diagrams contributing to the action of the instantonnucleus configuration. These diagrams are of the order of $\rho^{4}$ in the instanton size and involve only one 
interacting nucleon, that is only one power of $\alpha_{s}^{2} A^{1 / 3} \lesssim 1$. First let us point out some two-loop diagrams which vanish or can be shown to be suppressed after a simple calculation. Those include the diagrams where only one gluon interacts with the nucleus and the diagrams with the vertices of the type introduced in Fig. 11 C. For instance the graph in Fig. 2A vanishes for the same reason as the graph in Fig. 11C: it is proportional to $f^{a b c} \delta^{a b}=0$. All the diagrams generated by including all other possible connections of the extra gluon in Fig. 2A to the gluon and quark lines vanish for similar reasons of simple color algebra. The case when the gluon connects to the instanton via the instanton-two gluon vertex is a little different. To estimate the diagram in Fig. $2 \mathrm{~B}$ we have to make use of the fact that the nucleus is moving very fast in the light cone "+" direction. Then the gluon propagators for the exchanged gluons give $g_{\mu-} g_{\nu-}$ in covariant gauge. The contribution of the graph in Fig. $2 \mathrm{~B}$ is therefore proportional to

$$
g_{\mu-} g_{\nu-} \bar{\eta}_{d \mu \rho}^{M} R^{d d^{\prime}} f^{a c d^{\prime}} \eta_{e \nu \rho}^{M} R^{e e^{\prime}} f^{b c e^{\prime}} \delta^{a b}=-3 N_{c} g_{--}=0 .
$$

The gluon labeled $\rho$ in Fig. $2 \mathrm{~B}$ could also connect the instanton-two gluon vertex to the other two gluon lines. These kind of diagrams involve one instanton-single gluon vertex and one instanton-double gluon vertex, as shown in Fig. 3. The diagrams in Fig. 3 can also be safely neglected for the following reasons. The graph in Fig. $3 \mathrm{~A}$ is zero since in covariant gauge its contribution is proportional to $\bar{\eta}_{a \mu \nu}^{M} g_{\mu-} g_{\nu-} \sim g_{--}=0$.

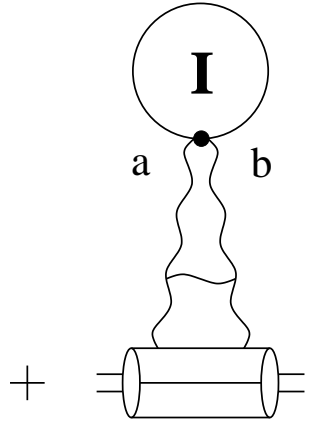

A

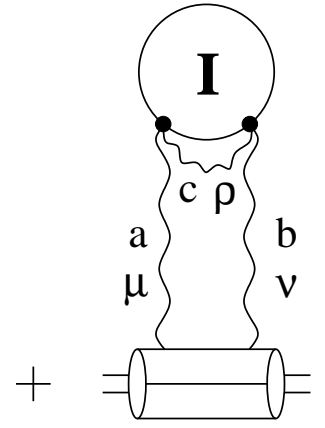

B

FIG. 2. Some of the two-loop graphs involving the instanton-two gluon vertex that vanish.

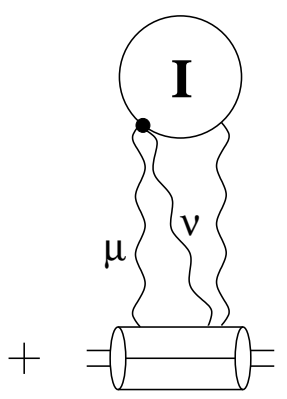

A

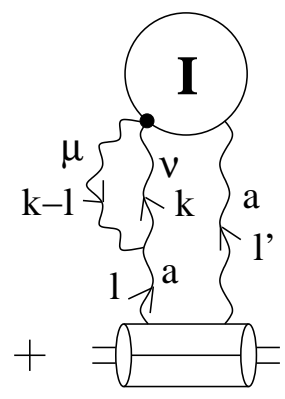

B

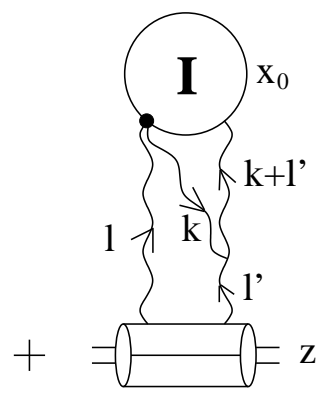

C

FIG. 3. A set of the two-loop graphs involving one instanton-double gluon vertex and one instanton-single gluon vertex that vanish.

To prove that the contribution of the diagram in Fig. $3 \mathrm{~B}$ is zero one has to add to it the diagram where the lines corresponding to $l$ and $l^{\prime}$ gluons connect to the nucleon in the opposite order (crossed diagram), which similarly to how in was shown in Eq. (17) for the graph of Fig. 11B would give us two delta functions $\delta\left(l_{-}\right) \delta\left(l_{-}^{\prime}\right)$. Thus one can show that diagram of Fig. $3 \mathrm{~B}$ is proportional to 


$$
\begin{gathered}
\bar{\eta}_{a \mu \nu}^{M}\left[(k-2 l)_{\nu} g_{\mu-}+(-2 k+l)_{-} g_{\mu \nu}+(l+k)_{\mu} g_{\nu-}\right] \eta_{a-\rho}^{M} l_{\rho}^{\prime} \delta\left(l_{-}\right) \delta\left(l_{-}^{\prime}\right)= \\
=-3 \bar{\eta}_{a-\nu}^{M} l_{\nu} \eta_{a-\rho}^{M} l_{\rho}^{\prime} \delta\left(l_{-}\right) \delta\left(l_{-}^{\prime}\right)=3 l_{-} l_{-}^{\prime} \delta\left(l_{-}\right) \delta\left(l_{-}^{\prime}\right)=0 .
\end{gathered}
$$

Finally one can show that in the eikonal approximation employed here the contribution of the graph in Fig. $3 \mathrm{C}$ is independent of $l_{+}$. Therefore the integral over $l_{+}$in that diagram yields us with [6]

$$
\int_{-\infty}^{\infty} \frac{d l_{+}}{2 \pi} e^{-i l_{+}\left(x_{0--} z_{-}\right)}=\delta\left(x_{0-}-z_{-}\right)
$$

where $x_{0}$ is the position of the instanton and $z_{-}$is the (frozen) light cone coordinate of the interacting quark in the nucleon. Eq. (20) requires that the nucleon interacting with the instanton at point $x_{0}$ should have the same light cone coordinate as the instanton. But this would not allow us to sum over all nucleons situated at different light cone coordinates at the given impact parameter to obtain the enhancement of this diagram by the powers of atomic number $A^{1 / 3}$ [6, 8, 35]. In other words the diagram of Fig. 3C would not be resumming powers of the parameter $\alpha_{s}^{2} A^{1 / 3}$, it would have the factor of $\alpha_{s}^{2}$ without any $A^{1 / 3}$ enhancement. Thus it is zero at the leading order in $A^{1 / 3}$.
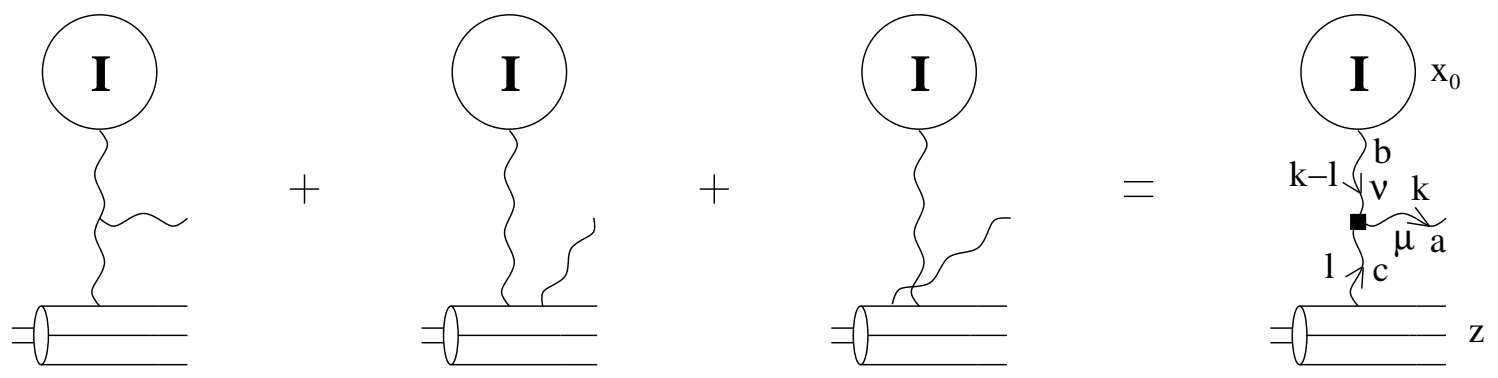

FIG. 4. Definition of the effective triple gluon vertex in $\partial_{\mu} A_{\mu}=0$ covariant gauge.

To start analyzing the diagrams with two instanton-single gluon vertices let us define an effective triple gluon vertex as shown in Fig. A. All the diagrams on the left of Fig. 1 are taken in covariant gauge with the gluons' light cone momenta being much smaller than the quarks light cone momentum. The graphs in Fig. 目 correspond to the first non-trivial perturbative contribution to the combined field of the instanton-nucleus configuration. An analogy could be drawn with the Lipatov vertex being the first non-trivial contribution to the field of two colliding nuclei [35, 8,38$]$. After a simple calculation one can see that the lowest order field of the I-nucleus system is

$$
\begin{aligned}
& A_{\mu}^{a}(k)=g f^{a b c}\left(T^{c}\right) R^{b b^{\prime}} 4 \pi^{2} \rho^{2} \int \frac{d^{4} l}{(2 \pi)^{4}} e^{-i l \cdot\left(x_{0}-z\right)}(2 \pi) \delta\left(l_{-}\right) \frac{(k-l)_{\sigma}}{(k-l)^{2}+i \epsilon} \frac{1}{k^{2}+i \epsilon} \\
& \quad \times\left(-\frac{1}{\underline{l}^{2}} \bar{\eta}_{b^{\prime} \nu \sigma}^{M}\left[(k-2 l)_{\mu} g_{\nu-}+(l+k)_{\nu} g_{\mu-}-2 k_{-} g_{\mu \nu}\right]+\bar{\eta}_{b^{\prime}-\sigma}^{M} g_{\mu-\frac{1}{k_{-}}}\right)
\end{aligned}
$$

where we Fourier transform over the momentum $l$ flowing between the nucleon at $z$ to the instanton at $x_{0}$. In Eq. (21) $\left(T^{c}\right)$ is the $S U(3)$ matrix in the color space of the interacting nucleon and $\underline{l}$ denotes the transverse component of momentum $l$.

To calculate the contribution to the action at the order $\rho^{4}$ of the field in Eq. (21) we have to consider the diagram depicted in Fig. 司A. After averaging in the color space of the nucleon below [6] the graph in Fig. 罒A yields us with

$$
i S_{A}=\frac{1}{2} \frac{g^{2}}{2}\left(4 \pi^{2} \rho^{2}\right)^{2} \int \frac{d^{4} k}{(2 \pi)^{4}} \frac{d^{4} l}{(2 \pi)^{4}} \frac{d^{4} l^{\prime}}{(2 \pi)^{4}} e^{-i l \cdot\left(x_{0}-z\right)-i l^{\prime} \cdot\left(x_{0}-z\right)}(2 \pi) \delta\left(l_{-}\right)(2 \pi) \delta\left(l_{-}^{\prime}\right) \frac{-i}{k^{2}+i \epsilon}
$$




$$
\begin{gathered}
\times \frac{(k-l)_{\sigma}}{(k-l)^{2}+i \epsilon} \frac{\left(k+l^{\prime}\right)_{\sigma^{\prime}}}{\left(k+l^{\prime}\right)^{2}-i \epsilon}\left(\frac{1}{\underline{l}^{2}}\left[-\bar{\eta}_{b-\sigma}^{M}(k-2 l)_{\mu}-\bar{\eta}_{b \nu \sigma}^{M}(l+k)_{\nu} g_{\mu-}+\bar{\eta}_{b \mu \sigma}^{M} 2 k_{-}\right]+\bar{\eta}_{b-\sigma}^{M} g_{\mu-} \frac{1}{k_{-}}\right) \\
\times\left(\frac{1}{\underline{l}^{\prime 2}}\left[-\eta_{b-\sigma^{\prime}}^{M}\left(k+2 l^{\prime}\right)_{\mu}-\eta_{b \nu^{\prime} \sigma^{\prime}}^{M}\left(k-l^{\prime}\right)_{\nu^{\prime}} g_{\mu-}+\eta_{b \mu \sigma^{\prime}}^{M} 2 k_{-}\right]+\eta_{b-\sigma^{\prime}}^{M} g_{\mu-} \frac{1}{k_{-}}\right)
\end{gathered}
$$

where the field on the right hand side of Fig. $5 \mathrm{~A}$ is complex conjugate to the field on the left hand side. The first factor of $1 / 2$ in Eq. (22) is the symmetry factor while the second factor of $1 / 2$ arises after averaging over colors in the nucleon. Simplifying the expression in Eq. (22) by employing the algebra of 't Hooft symbols [19] we obtain

$$
\begin{aligned}
& i S_{A}=4 \pi^{4} \rho^{4} g^{2} \int \frac{d^{4} k}{(2 \pi)^{4}} \frac{d^{4} l}{(2 \pi)^{4}} \frac{d^{4} l^{\prime}}{(2 \pi)^{4}} e^{-i l \cdot\left(x_{0}-z\right)-i l^{\prime} \cdot\left(x_{0}-z\right)}(2 \pi) \delta\left(l_{-}\right)(2 \pi) \delta\left(l_{-}^{\prime}\right) \frac{-i}{k^{2}+i \epsilon} \\
& \times \frac{1}{(k-l)^{2}+i \epsilon} \frac{1}{\left(k+l^{\prime}\right)^{2}-i \epsilon} \frac{k_{-}^{2}}{\underline{l}^{2} \underline{l}^{2}}\left[-13 k^{2}+14 k \cdot l-14 k \cdot l^{\prime}+24 l \cdot l^{\prime}-5 \underline{l}^{2}-5 \underline{l}^{\prime 2}\right] .
\end{aligned}
$$

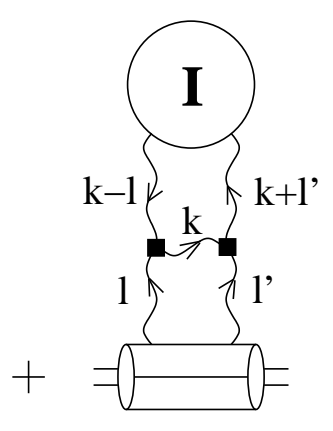

A

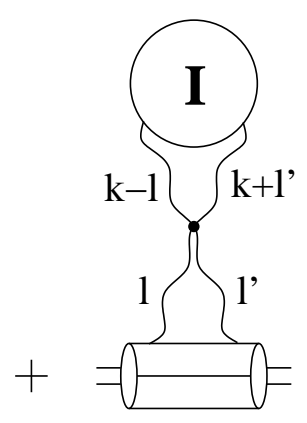

B

FIG. 5. Diagrams with two instanton-single gluon vertices that cancel each other at the leading order in $A$.

To average over the nuclear wave function one has to average over the positions of all the quarks in the nucleons and nucleons in the nucleus and sum over all the nucleons and quarks in them [6]. This could be summarized by the following operation

$$
\sum_{\text {nucleons val. quarks }} \sum_{S_{\perp} 2 b_{-}} \int \frac{d^{2} z d z_{-}}{S_{0}}
$$

which has to be applied to Eq. (23). For simplicity we consider a cylindrical nucleus with cross sectional area $S_{\perp}$ and the longitudinal extent of $2 b_{-}$in the infinite momentum frame. Applying Eq. (24) to Eq. (23) yields for a quarkonium nucleus of [6,36]

$$
\begin{aligned}
& i\left\langle S_{A}\right\rangle=-i 4 \pi^{4} \rho^{4} g^{2} \frac{2 A}{S_{\perp}} \int \frac{d^{4} k}{(2 \pi)^{4}} \frac{d^{2} l}{(2 \pi)^{2}} \frac{d l_{+} d l_{+}^{\prime}}{(2 \pi)^{2}} \frac{e^{-i l_{+} x_{0-}-i l_{+}^{\prime} x_{0-}}}{k^{2}\left[(k-l)^{2}+i \epsilon\right]\left[\left(k+l^{\prime}\right)^{2}-i \epsilon\right]} \frac{k_{-}^{2}}{\left(\underline{l}^{2}\right)^{2}} \\
& \times\left[-13 k^{2}+14 k \cdot l-14 k \cdot l^{\prime}+14 \underline{l}^{2}\right] .
\end{aligned}
$$

Performing similar calculations for the diagram in Fig. $5 \mathrm{~B}$ we obtain 


$$
i\left\langle S_{B}\right\rangle=i 4 \pi^{4} \rho^{4} g^{2} \frac{2 A}{S_{\perp}} \int \frac{d^{4} k}{(2 \pi)^{4}} \frac{d^{2} l}{(2 \pi)^{2}} \frac{d l_{+} d l_{+}^{\prime}}{(2 \pi)^{2}} \frac{e^{-i l_{+} x_{0-}-i l_{+}^{\prime} x_{0-}}}{\left[(k-l)^{2}+i \epsilon\right]\left[\left(k+l^{\prime}\right)^{2}-i \epsilon\right]} \frac{k_{-}^{2}}{\left(\underline{l}^{2}\right)^{2}} .
$$

Adding up the contributions of Eqs. (25) and (26) we write

$$
\begin{aligned}
i\left\langle S_{A+B}\right\rangle=-i 4 \pi^{4} \rho^{4} g^{2} \frac{2 A}{S_{\perp}} & \int \frac{d^{4} k}{(2 \pi)^{4}} \frac{d^{2} l}{(2 \pi)^{2}} \frac{d l_{+} d l_{+}^{\prime}}{(2 \pi)^{2}} \frac{e^{-i l_{+} x_{0-}-i l_{+}^{\prime} x_{0-}}}{k^{2}\left[(k-l)^{2}+i \epsilon\right]\left[\left(k+l^{\prime}\right)^{2}-i \epsilon\right]} \frac{k_{-}^{2}}{\left(\underline{l}^{2}\right)^{2}} \\
& \times(-7)\left[(k-l)^{2}+\left(k+l^{\prime}\right)^{2}\right] .
\end{aligned}
$$

If one wants to perform the $l_{+}$and $l_{+}^{\prime}$ integrals in Eq. (27) one has to pick up the poles given by the $(k-l)^{2}$ and $\left(k+l^{\prime}\right)^{2}$ denominators. However each of the terms in the square brackets at the end of Eq. (27) cancels one of these denominators making the expression in Eq. (27) zero for any non-zero $x_{0-}$. Thus the diagrams in Fig. 5 cancel each other and give zero at the leading powers in $A$.

That way we have shown that even at one loop order the field of a large nucleus does not affect the instanton distribution

$$
n_{\text {sat }}^{A}(\rho)=n_{0}(\rho) .
$$

The physical reason behind this result of our calculation is the following. We have seen in Sect. IIIA that for a purely classical field the only non-zero component of the field strength tensor is $G_{+\perp}^{W W a}$ [6], [], which leads to $G^{2}=G \tilde{G}=0$ and due to Eq. (14) the field does not affect the instanton size distribution. The diagrams we have analyzed above, especially the graphs in Fig. f correspond to including one rung of the QCD evolution in energy. (We were interested in the real part of the diagrams and did not get the factor of $\ln 1 / x$ from them.) Recently, in [10,12] a picture of the evolution in $\ln 1 / x$ has been developed which represents the quantum evolution as a series of classical emissions. At each step of the evolution the existing partons act as classical sources of color charge and emit a gluon. The gluon then gets incorporated into the source and acts as a color charge emitting gluons in the subsequent steps of the evolution?. This tells us that if we look at the gluon field generated by any number of evolution steps it would always look like a classical field emitted off some complicated (evolved) source moving in the "+" direction and therefore has only $G_{+\perp}^{W W a}$ non-zero component of the field strength tensor. Thus from Eq. (14) we derive that this field can not change the instanton density. Multiple rescatterings, i.e., higher powers of $\alpha_{s}^{2} A^{1 / 3}$ would not help to obtain a non-zero effect since $G^{2}=G \tilde{G}=0$ for the full non-Abelian Weizsäcker-Williams field of a large nucleus which includes the effects of all the multiple rescatterings in it 6 [ 8 ].

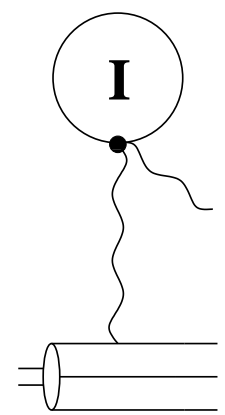

FIG. 6. Last diagram which along with the graphs in Fig. 1 contributes to the topologically non-trivial classical field of a large nucleus at the lowest order.

\footnotetext{
${ }^{2}$ This picture is based on the achieved long ago understanding that the appearance of $\ln (1 / x)$ for each emitted gluon reflects the fact that this gluon lives much longer light cone time than all gluons emitted after it but much shorter time than gluons emitted before it 47.
} 
Since the field of a single nucleus does not suppress instantons it would be interesting to construct a solution of the Yang-Mills equations of motion with the nuclear source carrying some non-zero topological charge. The effective action of Eq. (5) allows us to do that perturbatively. The lowest order field is just a direct sum of the fields of the instanton and the nucleus. At the lowest non-trivial order in $\rho^{2}$ and $g$ almost all the field is given by the diagrams contributing to the effective vertex of Fig. 1 and is written down in Eq. (21). Another diagram which contributes to the field at this lowest order is shown in Fig. 6 and involves and instanton-double gluon vertex.

Adding the contribution of the diagram from Fig. 6 to the contribution of graphs from Fig. 1 we obtain the following expression for the total field of the instanton-nucleus configuration at the lowest order in momentum space

$$
\begin{gathered}
A_{\mu}^{a}(k)=g f^{a b c}\left(T^{c}\right) R^{b b^{\prime}} 4 \pi^{2} \rho^{2} \int \frac{d^{4} l}{(2 \pi)^{4}} e^{-i l \cdot\left(x_{0}-z\right)}(2 \pi) \delta\left(l_{-}\right) \frac{1}{k^{2}+i \epsilon}\left[\frac{(k-l)_{\sigma}}{(k-l)^{2}+i \epsilon}\right. \\
\left.\times\left(-\frac{1}{\underline{l}^{2}} \bar{\eta}_{b^{\prime} \nu \sigma}^{M}\left[(k-2 l)_{\mu} g_{\nu-}+(l+k)_{\nu} g_{\mu-}-2 k_{-} g_{\mu \nu}\right]+\bar{\eta}_{b^{\prime}-\sigma}^{M} g_{\mu-} \frac{1}{k_{-}}\right)+\frac{i}{\underline{l}^{2}} \bar{\eta}_{b^{\prime} \mu-}^{M}\right] .
\end{gathered}
$$

To summarize the results of this section we once again point out that the saturation effects fail to either enhance or suppress instantons in a single hadron or nucleus (Eq. (28)), which makes possible the existence of a classical solution for the gluon field of the nucleus carrying a non-zero topological charge, a lowest order expression for which is given by Eq. (29) for the case of unit topological charge. Now that we have demonstrated the formalism for calculating the effects of a background classical field on the instanton distribution we continue by considering the case of hadronic or nuclear collisions.

\section{HADRONIC AND NUCLEAR COLLISIONS}

It was shown in 35 38] that the gluon production in the central rapidity region of a heavy ion collision at very high energies is dominated by the classical gluonic field produced by two colliding nuclei. The colliding nuclei could be visualized as ensembles of point color charges moving without any deflection through each other along the light cone [35], similarly to the case of a single nucleus in McLerran-Venugopalan model 顿. As the nuclei pass through each other the color charges in each nucleus get rotated in color space by the field of another nucleus, which leads to a bremsstrahlung emission of gluons off these charges [36]. The gluons then subsequently multiply rescatter in the background fields of both nuclei before being produced [38].

The produced classical field is boost invariant and fills the whole region between the nuclei moving apart after the collision [35] 38. The field is produced at all the impact parameters where the collision happens. Thus for central collisions the classical field covers the whole nuclear cross sectional area in the transverse direction $S_{\perp}$. In the longitudinal direction the typical gluon production time is of the order of $2 k_{+} / \underline{k}^{2}$. Since for classically produced gluons $|\underline{k}| \sim Q_{s}$ and $k_{+} \sim Q_{s}$ [38, 41, 53] the typical longitudinal time is of the order of $1 / Q_{s}$. This translates into the typical thickness in the $z$-direction of the region in which the classical fields are produced being of the order of $l \sim 1 / Q_{s}$. As was argues by Baier et al in [41] even though the classical field, as a leading order in $\alpha_{s}$ term, would still exist at later times the thermalization effects would become important there significantly modifying the distribution of produced gluons. In our effective lagrangian approach we consider relatively small instantons with sizes $\rho \ll l \sim 1 / Q_{s}$ (see Eq. (15)) which might easily appear in the spatial region described above at times of the order $\tau \sim 1 / Q_{s}$. For such small instantons it is reasonable to consider the problem of instantons in the classical field produced in a nuclear collision before thermalization effects became important.

To estimate the effect of this classical gluon field produced in mid-rapidity on instantons one has to calculate the field strength tensor of this field and substitute it into Eq. (14) averaging over the wave functions of both colliding nuclei. An analytical expression for the classical field exists only at the lowest non-trivial order 35, 36]. It is possible to calculate the multiplicity distribution of the produced particles analytically to all orders in the background field without deriving an explicit expression for the field [38]. Numerical results exist for the field and multiplicity distribution [37]. We begin by calculating the field strength tensor at the lowest order corresponding to the case when $\alpha_{s}^{2} A_{1}^{1 / 3} \lesssim 1$ and $\alpha_{s}^{2} A_{2}^{1 / 3} \lesssim 1$, i.e., the weak 
field case. $A_{1}$ and $A_{2}$ are the atomic numbers of the colliding nuclei. In this case only one nucleon from each nucleus contributes to the classical field. We will later generalize our result to the strong field case of $\alpha_{s}^{2} A_{1}^{1 / 3} \sim 1$ and $\alpha_{s}^{2} A_{2}^{1 / 3} \sim 1$

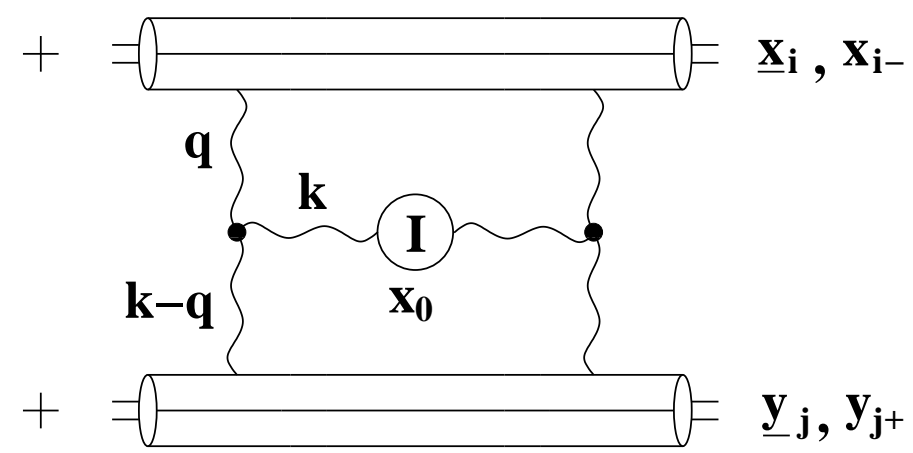

FIG. 7. Instanton in the background gluon field of colliding nuclei, which is taken at the lowest non-trivial order in $g$. Thick dots represent Lipatov vertices.

The lowest order classical gluon field of two colliding nucleons $i$ and $j$ having coordinates $\underline{x}_{i}, x_{i-}$ and $\underline{y}_{j}$, $y_{j+}$ correspondingly in covariant gauge $\partial \cdot A=0$ is given by [36]

$$
A_{\mu}^{a}(x)=-i \int \frac{d^{4} k}{(2 \pi)^{4}} \frac{e^{-i k \cdot x}}{k^{2}+i \epsilon k_{0}} \int d^{2} q \frac{g^{3}}{(2 \pi)^{2}} f^{a b c}\left(T_{i}^{b}\right)\left(\tilde{T}_{j}^{c}\right) e^{\left.i\left[k_{+} x_{i-}+k_{-} y_{j+}-\underline{k} \cdot \underline{y}_{j}-\underline{q}^{\prime} \cdot \underline{x}_{i}-\underline{y}_{j}\right)\right]} \frac{C_{\mu}(k, \underline{q})}{\underline{q}^{2}(\underline{k}-\underline{q})^{2}}
$$

where $C_{\mu}(k, \underline{q})$ is the Lipatov vertex 17$]$

$$
C_{\mu}(k, \underline{q})=\left(\frac{\underline{q}^{2}}{k_{-}+i \epsilon}-k_{+},-\frac{(\underline{k}-\underline{q})^{2}}{k_{+}+i \epsilon}+k_{-}, 2 \underline{q}-\underline{k}\right) .
$$

In Eq. (31) the four-vector $C_{\mu}$ is shown in terms of its components in the $(+,-, \perp)$ form. $\left(T_{i}^{b}\right)$ and $\left(\tilde{T}_{j}^{c}\right)$ in Eq. (30) are matrices in the color spaces of the colliding nucleons [6, 36]. Regularization of the gluon propagator $k^{2}$ in Eq. (30) corresponds to using retarded Green function and is used to insure casuality of the classical field: it can be non-zero only in the forward light cone [36].

Let us start by calculating the averaged value of the square of the field strength tensor $\left\langle\left[G_{\mu \nu}^{a}\left(x_{0}\right)\right]^{2}\right\rangle$ to be used in Eq. (14) at the lowest order in $\alpha_{s}$. One can easily see that at the lowest order in the coupling $g$ only the Abelian part of $G_{\mu \nu}^{a}$ would contribute. We are interested only in the $\left\langle\left[G_{\mu \nu}^{a}\left(x_{0}\right)\right]^{2}\right\rangle$ in the forward light cone in the central rapidity region. Thus we do not need to include the effects of the lowest order fields of each of the nuclei which give non-zero $G_{\mu \nu}^{a}$ only on the light cone (at $x_{+}=0$ and/or $x_{-}=0$ ). The diagram that we need to calculate is shown in Fig. 7 and corresponds to the lowest order gluon field $\left(\right.$ order $\left.g^{3}\right)$ interacting with the instanton at the lowest order in $\rho^{2}$ (order $\left.\rho^{4}\right)$. Blobs in Fig. 7 denote Lipatov vertices.

The contribution of the diagram in Fig. 7 is

$$
\left\langle G_{\mu \nu}^{a}\left(x_{0}\right) G_{\mu \nu}^{a}\left(x_{0}\right)\right\rangle_{L O}=\left\langle\left[\partial_{\mu} A_{\nu}^{a}\left(x_{0}\right)-\partial_{\nu} A_{\mu}^{a}\left(x_{0}\right)\right]^{2}\right\rangle
$$

with $x_{0}$ the position of the instanton. Substituting the field of Eq. (30) into Eq. (32) and averaging over colors and positions of nucleons using Eq. (24) we obtain

$$
\begin{gathered}
\left\langle G_{\mu \nu}^{a}\left(x_{0}\right) G_{\mu \nu}^{a}\left(x_{0}\right)\right\rangle_{L O}=\frac{g^{6}}{(2 \pi)^{2}} \frac{A_{1} A_{2}}{S_{1 \perp} S_{2 \perp}} 2 C_{F} \int \frac{d^{4} k}{(2 \pi)^{4}} \frac{d k_{+}^{\prime} d k_{-}^{\prime}}{(2 \pi)^{2}} \frac{e^{-i k_{+} x_{0-}-i k_{-} x_{0+}-i k_{+}^{\prime} x_{0-}-i k_{-}^{\prime} x_{0+}}}{\left(k^{2}+i \epsilon k_{0}\right)\left(k^{\prime 2}+i \epsilon k_{0}^{\prime}\right)} \\
\times \int \frac{d^{2} q}{\left[\underline{q^{2}}(\underline{k}-\underline{q})^{2}\right]^{2}}\left[k_{\mu} C_{\nu}(k, \underline{q})-k_{\nu} C_{\mu}(k, \underline{q})\right]\left[k_{\mu}^{\prime} C_{\nu}\left(k^{\prime},-\underline{q}\right)-k_{\nu}^{\prime} C_{\mu}\left(k^{\prime},-\underline{q}\right)\right]
\end{gathered}
$$


where throughout Eq. (33) we imply that $\underline{k}^{\prime}=-\underline{k}$. In arriving at Eq. (33) we for simplicity assumed that the colliding nuclei are cylinders in the $z$ direction with cross sectional areas of $S_{1 \perp}$ and $S_{2 \perp}$ correspondingly. As was shown in [36] in the integration over the light cone components of $k$ and $k^{\prime}$ only the poles in the propagators $k^{2}$ and $k^{\prime 2}$ contribute. If one picks up the poles in the Lipatov vertices (see Eq. (31)) the resulting contribution would be non-zero only on the light cone [36]. However here we are interested in the central rapidity region only and that contribution would not be important to us. Thus only the gluon propagator poles would contribute in Eq. (33). Anticipating this we may estimate the value of the terms in the square brackets at the end of Eq. (33) employing the $k^{2}=0$ and $k^{\prime 2}=0$ conditions. After some lengthy algebra one ends up with

$$
\begin{gathered}
{\left.\left[k_{\mu} C_{\nu}(k, \underline{q})-k_{\nu} C_{\mu}(k, \underline{q})\right]\left[k_{\mu}^{\prime} C_{\nu}\left(k^{\prime},-\underline{q}\right)-k_{\nu}^{\prime} C_{\mu}\left(k^{\prime},-\underline{q}\right)\right]\right|_{k^{2}=k^{\prime 2}=0}=} \\
=\frac{8 k \cdot k^{\prime}}{\underline{k}^{2}}\left[-2(\underline{k} \cdot \underline{q})^{2}+\underline{k}^{2} \underline{q}^{2}+2(\underline{k} \cdot \underline{q}) \underline{q}^{2}-\left(\underline{q}^{2}\right)^{2}\right] .
\end{gathered}
$$

Substituting Eq. (34) into Eq. (33) yields

$$
\begin{gathered}
\left\langle G_{\mu \nu}^{a}\left(x_{0}\right) G_{\mu \nu}^{a}\left(x_{0}\right)\right\rangle_{L O}=\frac{g^{6}}{(2 \pi)^{2}} \frac{A_{1} A_{2}}{S_{1 \perp} S_{2 \perp}} 2 C_{F} \int \frac{d^{2} k d^{2} q}{(2 \pi)^{4}} \frac{8\left[-\underline{q}^{2}(\underline{k}-\underline{q})^{2}+2 \underline{k}^{2} \underline{q}^{2}-2(\underline{k} \cdot \underline{q})^{2}\right]}{\underline{k}^{2}\left[\underline{q}^{2}(\underline{k}-\underline{q})^{2}\right]^{2}} \\
\times \frac{d k_{+} d k_{-} d k_{+}^{\prime} d k_{-}^{\prime}}{(2 \pi)^{2}} \frac{e^{-i k_{+} x_{0-}-i k_{-} x_{0+}-i k_{+}^{\prime} x_{0-}-i k_{-}^{\prime} x_{0+}}}{\left(k^{2}+i \epsilon k_{0}\right)\left(k^{\prime 2}+i \epsilon k_{0}^{\prime}\right)} k \cdot k^{\prime} .
\end{gathered}
$$

One can readily check that

$$
\int d^{2} q \frac{\left[-\underline{q}^{2}(\underline{k}-\underline{q})^{2}+2 \underline{k}^{2} \underline{q}^{2}-2(\underline{k} \cdot \underline{q})^{2}\right]}{\left[\underline{q}^{2}(\underline{k}-\underline{q})^{2}\right]^{2}}=-\frac{\pi}{\underline{k}^{2}} .
$$

To obtain Eq. (36) it is easier to first integrate over the angles between $\underline{k}$ and $q$ after which the integration over $|\underline{q}|$ becomes trivial. Employing Eq. (36) in Eq. (35) we arrive at

$$
\begin{aligned}
& \left\langle G_{\mu \nu}^{a}\left(x_{0}\right) G_{\mu \nu}^{a}\left(x_{0}\right)\right\rangle_{L O}=-\frac{g^{6}}{(2 \pi)^{2}} \frac{A_{1} A_{2}}{S_{1 \perp} S_{2 \perp}} 2 C_{F} 8 \pi \int \frac{d^{2} k}{(2 \pi)^{4}\left(\underline{k}^{2}\right)^{2}} \\
& \times \frac{d k_{+} d k_{-} d k_{+}^{\prime} d k_{-}^{\prime}}{(2 \pi)^{2}} \frac{e^{-i k_{+} x_{0-}-i k_{-} x_{0+}-i k_{+}^{\prime} x_{0-}-i k_{-}^{\prime} x_{0+}}}{\left(k^{2}+i \epsilon k_{0}\right)\left(k^{\prime 2}+i \epsilon k_{0}^{\prime}\right)} k \cdot k^{\prime} .
\end{aligned}
$$

Evaluating the integral over the light cone components of $k$ and $k^{\prime}$ in Eq. (37) we note that the integral over $k_{\perp}$ in Eq. (37) is dominated by small transverse momenta $k_{\perp}$. Taking the $k_{\perp} \rightarrow 0$ limit of the longitudinal integral we obtain

$$
\int \frac{d k_{+} d k_{-} d k_{+}^{\prime} d k_{-}^{\prime}}{(2 \pi)^{2}} \frac{e^{-i k_{+} x_{0-}-i k_{-} x_{0+}-i k_{+}^{\prime} x_{0-}-i k_{-}^{\prime} x_{0+}}}{\left(k^{2}+i \epsilon k_{0}\right)\left(k^{\prime 2}+i \epsilon k_{0}^{\prime}\right)} k \cdot k^{\prime} \approx \frac{1}{\tau_{0}^{2}},
$$

where $\tau_{0}=\sqrt{2 x_{0+} x_{0-}}$ is the proper time. Here we made use of the fact that we are interested in instantons in the forward light cone $x_{0+}>0$ and $x_{0-}>0$. The approximation used in obtaining Eq. (38) is equivalent to taking the limit of early proper time, which more formally means $\tau_{0} Q_{s} \ll 1$. This approximation is not crucial for our approach and is made only to simplify the calculations.

Employing Eq. (38) in Eq. (37) yields

$$
\left\langle G_{\mu \nu}^{a}\left(x_{0}\right) G_{\mu \nu}^{a}\left(x_{0}\right)\right\rangle_{L O}=-\alpha_{s}^{3} \frac{A_{1} A_{2}}{S_{1 \perp} S_{2 \perp}} C_{F} \frac{16}{\pi^{2}} \frac{1}{\tau_{0}^{2}} \int \frac{d^{2} k}{\left(\underline{k}^{2}\right)^{2}} .
$$


Before we discuss the issue of regularization of the divergent integral over transverse momentum in Eq. (39) let us first evaluate the other correlator in the exponent of Eq. (14). The lowest order field of Eq. (30) yields after averaging over nucleons similarly to Eq. (33)

$$
\begin{aligned}
\left\langle G_{\mu \nu}^{a}\left(x_{0}\right) \tilde{G}_{\mu \nu}^{a}\left(x_{0}\right)\right\rangle_{L O} & =\frac{g^{6}}{(2 \pi)^{2}} \frac{A_{1} A_{2}}{S_{1 \perp} S_{2 \perp}} 2 C_{F} \int \frac{d^{4} k}{(2 \pi)^{4}} \frac{d k_{+}^{\prime} d k_{-}^{\prime}}{(2 \pi)^{2}} \frac{e^{-i k_{+} x_{0-}-i k_{-} x_{0+}-i k_{+}^{\prime} x_{0-}-i k_{-}^{\prime} x_{0+}}}{\left(k^{2}+i \epsilon k_{0}\right)\left(k^{\prime 2}+i \epsilon k_{0}^{\prime}\right)} \\
& \times \int \frac{d^{2} q}{\left[\underline{q}^{2}(\underline{k}-\underline{q})^{2}\right]^{2}} 2 \epsilon_{\mu \nu \rho \sigma} k_{\mu} C_{\nu}(k, \underline{q}) k_{\rho}^{\prime} C_{\sigma}\left(k^{\prime},-\underline{q}\right)
\end{aligned}
$$

where again $\underline{k}^{\prime}=-\underline{k}$. After some simple algebra one obtains

$$
\left.\epsilon_{\mu \nu \rho \sigma} k_{\mu} C_{\nu}(k, \underline{q}) k_{\rho}^{\prime} C_{\sigma}\left(k^{\prime},-\underline{q}\right)\right|_{k^{2}=k^{\prime 2}=0}=8 \epsilon_{\mu \nu} k_{\mu}^{\perp} q_{\nu}^{\perp} \underline{q} \cdot(\underline{k}-\underline{q}),
$$

where we have again anticipated that the integration over the longitudinal momenta has to pick up the poles of the gluon propagators giving $k^{2}=k^{\prime 2}=0$ similarly to the way we used it in obtaining Eq. (34). Eq. (41) together with Eq. (40) gives

$$
\left\langle G_{\mu \nu}^{a}\left(x_{0}\right) \tilde{G}_{\mu \nu}^{a}\left(x_{0}\right)\right\rangle_{L O} \sim \int \frac{d^{2} q}{\left[\underline{q}^{2}(\underline{k}-\underline{q})^{2}\right]^{2}} \epsilon_{\mu \nu} k_{\mu}^{\perp} q_{\nu}^{\perp} \underline{q} \cdot(\underline{k}-\underline{q})=0 .
$$

The fact that the integral over $q_{\perp}$ in Eq. 42 is zero could be seen by changing variables $\underline{q} \rightarrow \underline{k}-\underline{q}$ which would demonstrate that the integral is equal to its negative. We conclude therefore that at this lowest non-trivial order in the coupling constant

$$
\left\langle G_{\mu \nu}^{a}\left(x_{0}\right) \tilde{G}_{\mu \nu}^{a}\left(x_{0}\right)\right\rangle_{L O}=0 .
$$

We think that (43) is a general result required by the symmetry of the problem. Indeed, since the topological charge (43) determines the net helicity of the system, and on the average the net helicity generated in the collision is equal to zero, the expectation value of the topological charge should vanish. This does not, however, imply a zero dispersion in the topological charge distribution. The event-by-event fluctuations of topological charge can exist, and would induce parity-odd correlations in the multiparticle production [52].

The only non-zero correlator for the classical gluon field produced in a hadronic or nuclear collision is thus $\left\langle G_{\mu \nu}^{a}\left(x_{0}\right) G_{\mu \nu}^{a}\left(x_{0}\right)\right\rangle$. The correlator in Eq. (39) has an infrared-divergent integral in it. The divergence is similar to the infrared singularity present in multiplicity distribution of the produced gluons given by the lowest order perturbative diagram in Fig. 1 (without the instanton). As was argued in [35] 38,53] multiple rescatterings would regularize the integral by effectively inserting an infrared cutoff in the $k_{\perp}$ integral in Eq. (39) which would be proportional to the saturation scale $Q_{s}$. Unfortunately we do not know exactly the coefficient of proportionality between this effective cutoff and $Q_{s}$. At the same time this coefficient would be very important for evaluating the integral in Eq. (39).

There are several ways to regularize the integral in Eq. (39). Saturation effects which regularize the integral in the infrared could be included in the classical (multiple rescattering) approximation ,4,63 8, 38, 53, or in a more realistic way incorporating the effects of quantum evolution in energy [1,2,5,5, 16,54. The latter way involves resummation of leading logarithms of energy (i.e. powers of $\alpha_{s} \ln 1 / x$ ) which arise from developing additional soft partons in the nuclear wave functions that will be produced in the collision. However, as was argued in 12,55 the effect of this quantum evolution is only to produce sources of color charge off which the classical field would be emitted. This statement was quantified in [12, 13,54, for a single nucleus. Unfortunately similar analysis has not been carried out for the gluon production in nuclear collisions. Thus we will restrict ourselves to the case of classical field emitted off the valence quarks in the nucleons of the colliding nuclei 35,36 . The quantum evolution will be suppressed implying that $\alpha_{s} \ln 1 / x \lesssim 1$ and no extra gluons are produced. Of course generalization of Eq. (39) to include the full effect of saturation strictly speaking does not reduce to the problem of classical gluon production of [35, 36] due to the nonAbelian nature of $G_{\mu \nu}^{a}$ which leads to appearance of three- $A_{\mu}$ and four- $A_{\mu}$ correlators in the full correlator $\left\langle G_{\mu \nu}^{a}\left(x_{0}\right) \tilde{G}_{\mu \nu}^{a}\left(x_{0}\right)\right\rangle$. Thus the calculations presented below should be understood as an estimate of what the exact answer should be in the strong field case. 
We are going to conjecture the following procedure of generalizing the result of Eq. (39) to the strong field case of $\alpha_{s}^{2} A_{1}^{1 / 3} \sim 1$ and $\alpha_{s}^{2} A_{2}^{1 / 3} \sim 1$. Let us first note that at the same order in the coupling constant as was employed in Eq. (39) the multiplicity of gluons produced in a collision of two quarkonium nuclei is given by [36]

$$
\frac{d N}{d^{2} b d y}=\alpha_{s}^{3} \frac{A_{1} A_{2}}{S_{1 \perp} S_{2 \perp}} C_{F} \frac{32}{\pi} \int \frac{d^{2} k}{\left(\underline{k}^{2}\right)^{2}} \ln \frac{k_{\perp}}{\Lambda}
$$

with $\Lambda$ some infrared cutoff, $b$ the impact parameter and $y$ rapidity of the produced gluon. As one can see the expression in Eq. (44) has an infrared singularity similar to the one in Eq. (39). Assuming that both singularities get regulated in a similar fashion we write with logarithmic accuracy

$$
\left\langle G_{\mu \nu}^{a}\left(x_{0}\right) G_{\mu \nu}^{a}\left(x_{0}\right)\right\rangle \approx-\frac{1}{2 \pi \tau_{0}^{2}} \frac{d N}{d^{2} b d y} .
$$

We thus suppose that Eq. (45) holds at all higher orders after inclusion of multiple rescatterings and is independent of whether we take a quarkonium model of the nuclei or realistic heavy ions. It has been conjectured in [53] that the multiplicity of the produced gluons including all higher order multiple rescattering effects is proportional to the multiplicity of gluons in the nuclear wave function before the collision so that for the case of two identical colliding cylindrical nuclei

$$
\frac{d N}{d^{2} b d y}=c \frac{C_{F} Q_{s}^{2}}{\alpha_{s} 2 \pi^{2}}
$$

Eq. (46) has been written in [53] with logarithmic accuracy. The proportionality coefficient in Eq. (46) has been estimated numerically to be $c=1.29 \pm 0.09$ [37]. An analytical calculation of [38] gives $c \approx 2 \ln 2 \approx 1.39$ and RHIC data seems to suggest that $c=1.23 \pm 0.20$ [45]. Inserting Eq. (46) into Eq. (45) yields

$$
\left\langle G_{\mu \nu}^{a}\left(x_{0}\right) G_{\mu \nu}^{a}\left(x_{0}\right)\right\rangle \approx-c \frac{C_{F} Q_{s}^{2}}{4 \pi^{3} \alpha_{s} \tau_{0}^{2}},
$$

which, after being substituted in Eq. (14) gives the distribution of instantons

$$
n_{\text {sat }}^{A A}(\rho)=n_{0}(\rho) \exp \left(-\frac{c \rho^{4} Q_{s}^{4}}{8 \alpha_{s}^{2} N_{c}\left(Q_{s} \tau_{0}\right)^{2}}\right) .
$$

Eq. (48) is our main result. It shows that large size instantons are suppressed by the strong classical fields generated in the nuclear collision. As was argued in [41] classical fields are a good description of the produced gluonic medium only at the early times $\tau_{0} Q_{s} \lesssim 1$. Thus for $\tau_{0} \sim 1 / Q_{s}$ the suppression starts parametrically when $\rho^{2} Q_{s}^{2} \sim \alpha_{s} \ll 1$, that is our main assumption of small instantons stated in Eq. (15) is still valid.

Three remarks are in order here. First let us note that the instanton suppression of Eq. (48) results from Eq. (14) applied to the case of nuclear collisions. Eq. (14) was originally derived for the instanton distribution in the background of the color field generated by QCD vacuum fluctuations as considered in [20,24]. By definition of the physical vacuum the energy density in it is negative with respect to perturbative vacuum. Thus for the field of vacuum fluctuations $\left\langle\theta_{\mu}^{\mu}\right\rangle<0$. Recalling that for QCD in the chiral limit of massless quarks

$$
\left\langle\theta_{\mu}^{\mu}\right\rangle=-\frac{b g^{2}}{32 \pi^{2}}\left\langle G_{\mu \nu}^{a 2}\right\rangle
$$

we conclude that for vacuum fluctuations $\left\langle G_{\mu \nu}^{a 2}\right\rangle>0$. Therefore Eq. (14) with the background field of vacuum fluctuations having positive $\left\langle G_{\mu \nu}^{a 2}\right\rangle$ led the authors of [20,24] to conclude that the large size instantons are enhanced in QCD vacuum. The phenomenon is also referred to as instanton melting. The situation is very different for nuclear collisions. The energy density of the gluon matter produced in a heavy ion collision is positive with the trace of the energy-momentum tensor $\left\langle\theta_{\mu}^{\mu}\right\rangle>0$. With the help of Eq. (49) we conclude that produced gluon matter should have $\left\langle G_{\mu \nu}^{a 2}\right\rangle<0$, which agrees with Eq. (47). Thus Eq. (14) with negative $\left\langle G_{\mu \nu}^{a 2}\right\rangle$ leads to suppression of large size instantons in nuclear collisions as appears in Eq. (48). 
Secondly, it may appear that the instanton suppression of Eq. (48) contradicts the model of soft pomerons in hadronic collisions proposed by the authors in [31]. There each rung of the soft pomeron ladder was modeled by the instanton-induced transitions of two t-channel gluons into any number of s-channel gluons. If the instantons were suppressed so would be the effects described in 311. However the soft pomeron describes the total cross sections in the proton-proton scattering in the kinematical range of the Tevatron. There, even though the energies are high the colliding particles are protons $(A=1)$, not nuclei and unitarization effects are still weak if present at all. The corresponding saturation scale is presumably quite small, possibly being of the order of $\Lambda_{Q C D}$. Our suppression of Eq. (48) would be indistinguishable from the "usual" suppression of large size instantons in the instanton gas models of QCD vacuum [50] and therefore would not significantly alter the results of 31]. Alternatively one could say that the gluon fields generated in proton-proton collisions at Tevatron energies are not as strong as the fields produced in nuclear collisions at similar energies and thus can not introduce a strong suppression of instantons.

When the energy of the proton-proton collisions becomes extremely large saturation and unitarization effects begin to take place. As was argued in [1, 2. [4, 4,53 at sufficiently high energies the saturation scale even in proton-proton collisions would become large, much larger than $\Lambda_{Q C D}$, making most of the partons in the protons' wave functions perturbative. At these high energies the hard (BFKL) pomeron would be unitarized through multiple pomeron exchanges [5,96 12], which in fact would generate the large saturation scale $Q_{s}$. At the same time unitarization of the soft pomeron is not quite understood. There is a possibility that the soft pomeron unitarizes through multiple pomeron exchanges, similar to the hard pomeron. Here we would like to outline another possibility, inspired by the soft pomeron model of [31] and by the result of Eq. (48). The instanton-induced interactions leading to the soft pomeron behavior of the cross section would be suppressed in pp at high energies due to instanton suppression of Eq. (48). Thus the soft pomeron of [31] could be eliminated by the large perturbative saturation scale at extremely high energies and the cross sections would be dominated by hard perturbative interactions which are unitary.

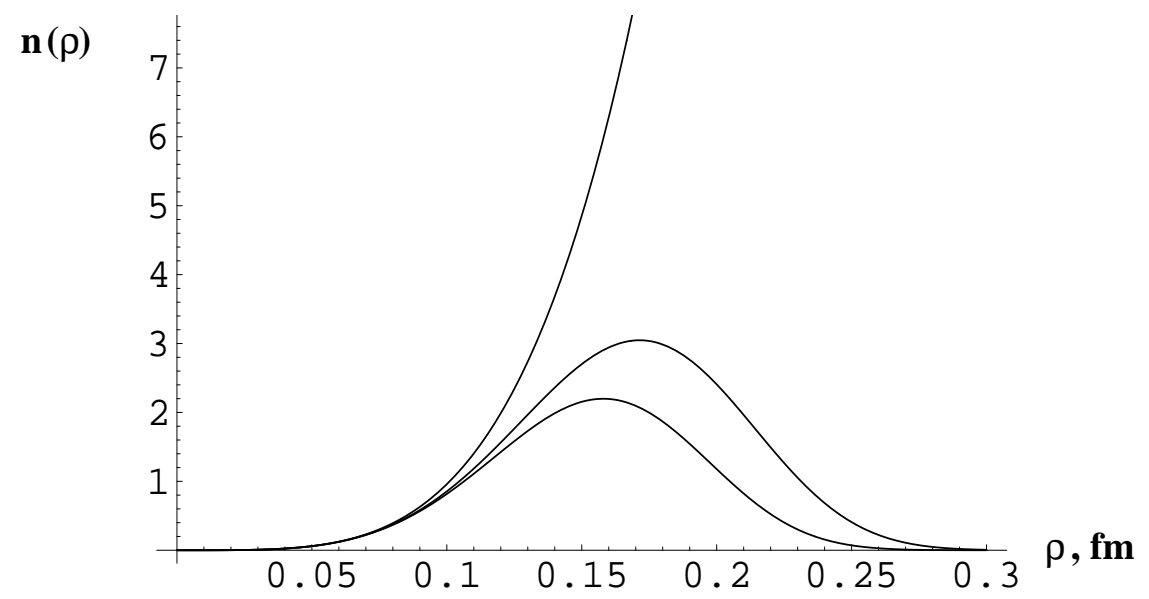

FIG. 8. Distributions of instanton sizes in vacuum for QCD with three light flavors (upper curve) versus the distribution of instanton sizes in the saturation environment produced by a collision of two identical nuclei for $c=1$ (middle curve) and $c=2 \ln 2$ (lower curve) with $Q_{s}^{2}=2 \mathrm{GeV}^{2}$ as estimated for RHIC at $\sqrt{s}=130 \mathrm{AGeV}$ in 45 .

Third we have to note that the suppression of Eq. (48) is different from the instanton suppressions at finite $T$ and $\mu$. There the power of the exponent is quadratic in $\rho$ (see Eqs. (11) and (3)) while in our case it is quadric in $\rho$. The difference is probably due to different physical mechanisms leading to instanton suppression. At high temperature or density the interactions of the gluons and quarks with each other generate a screening mass for gluons $m_{D}$. The gluoelectric fields are therefore screened at distances roughly of the order of $1 / m_{D}$, which leads to instanton suppression as discussed in the Introduction. In our case the gluon screening on the transverse scales of order $1 / Q_{s}$ is due to multiple rescatterings in the background classical field generated not by the other gluons but by the nuclei themselves. Therefore our expression in Eq. (48) is different from the high temperature and high density expressions in Eqs. (11) and (3).

In Fig. 目 we have plotted the perturbative distribution of instantons in vacuum $n_{0}(\rho)$ of Eq. (7) including 
three light flavors in the beta function $b=9$ (upper curve) together with the suppressed distributions of instantons in heavy ion collisions given by Eq. (48) for two different values of the "liberation coefficient" $c$ : the middle curve corresponds to $c=1$ and the lower curve corresponds to $c=2 \ln 2$. Saturation scale for the identical cylindrical nuclei was taken to be $Q_{s}^{2}=2 \mathrm{GeV}^{2}$ based on recent RHIC data 34, 45]. The strong coupling constant at this scale was approximated by $\alpha_{s}\left(Q_{s}\right) \approx 0.3$. The suppression curves are plotted for relatively late proper time $\tau_{0}=1 / Q_{s}$. Instanton suppression is even stronger for earlier times.

The integral of $n_{0}(\rho)$ over all $\rho$ gives the total instanton density at a given space-time point. Of course we can not integrate the perturbative size distribution of Eq. (7) over all values of $\rho$ since it diverges in the infrared. Instead we will employ the fit to lattice data for the instanton distribution in pure gluodynamics from [46]. The density of instantons given by this fit is $10.8 \mathrm{fm}^{-4}$. Integration of Eq. (48) with $n_{0}(\rho)$ given by the fit of [46] and with $Q_{s}^{2}=2 \mathrm{GeV}^{2}, c=2 \ln 2$ yields us with the instanton density at $\tau_{0}=1 / Q_{s}$ of approximately $0.007 \mathrm{fm}^{-4}$. The ratio of the two numbers gives the overall suppression of instantons density in the central rapidity region at RHIC to be 0.0006 . This estimate is made for the case of pure gluodynamics and the number will of course change once the effects of quarks are included. Nevertheless we still expect to have approximately three orders of magnitude suppression of instantons. Since the saturation scale increases with center of mass energy [1,9, 11, 14 we expect the suppression to get stronger for $\sqrt{s}=200 \mathrm{AGeV}$ at RHIC and for LHC energies. Numerical estimates of [15, 16 give the increase of $Q_{s}(x)$ for gold nuclei approximately by a factor of two for the LHC energy [15, 16]. Therefore the expected suppression will be considerably stronger.

We hope that our results open the possibility of a systematic theoretical investigation of topological effects in high energy nuclear collisions and introduce a different angle of looking at the problem of the interface between "hard" (perturbative) and "soft" (non-perturbative) interactions.

\section{ACKNOWLEDGMENTS}

We would like to thank Ian Balitsky, Dmitri Diakonov, Asher Gotsman, Uri Maor, Larry McLerran, John Negele, Victor Petrov, Rob Pisarski, Maxim Polyakov, Edward Shuryak and Larry Yaffe for many informative and stimulating discussions. The research of D.K. is supported by the U.S. Department of Energy under contract No. DE-AC02-98CH10886. The work of Yu.K. was supported in part by the U.S. Department of Energy under Grant No. DE-FG03-97ER41014. The research of Yu. K. and E. L. was sponsored in part by the BSF grant \# 9800276 and by Israeli Science Foundation, founded by the Israeli Academy of Science and Humanities.

[1] L. V. Gribov, E. M. Levin, and M. G. Ryskin, Phys. Rep. 100, 1 (1983); A.H. Mueller, J.-W. Qiu, Nucl. Phys. B268, 427 (1986).

[2] E.M. Levin and M.G Ryskin, Nucl. Phys. B304, 805 (1988); Sov. J. Nucl. Phys. 45, 150 (1987); 41, 300 (1985).

[3] A.H. Mueller, Nucl. Phys. B335, 115 (1990).

[4] L. McLerran and R. Venugopalan, Phys. Rev. D 49, 2233 (1994); 49, 3352 (1994); 50, 2225 (1994).

[5] A.L.Ayala, M.B.Gay Ducati, E.M.Levin, Nucl. Phys. B493, 305 (1997).

[6] Yu.V. Kovchegov, Phys. Rev. D 54, 5463 (1996); 55, 5445 (1997).

[7] J. Jalilian-Marian, A. Kovner, L. McLerran, and H. Weigert, Phys. Rev. D 55,5414 (1997).

[8] Yu. V. Kovchegov, A.H. Mueller, Nucl. Phys. B529, 451 (1998).

[9] Yu. V. Kovchegov, Phys. Rev. D 60, 034008 (1999); D 61, 074018 (2000).

[10] A.H. Mueller, Nucl. Phys. B415, 373 (1994); A.H. Mueller and B. Patel, Nucl. Phys. B425, 471 (1994); A.H. Mueller, Nucl. Phys. B437, 107 (1995); Z. Chen, A.H. Mueller, Nucl. Phys. B451, 579 (1995).

[11] I. I. Balitsky, Report No. hep-ph/9706411; Nucl. Phys. B463, 99 (1996); Phys. Rev. D 60, 014020 (1999).

[12] J. Jalilian-Marian, A. Kovner, A. Leonidov, and H. Weigert, Nucl. Phys. B 504, 415 (1997); Phys. Rev. D 59 014014 (1999); Phys. Rev. D59, 034007 (1999); J. Jalilian-Marian, A. Kovner, and H. Weigert, Phys. Rev. D 59014015 (1999). 
[13] A. Kovner, J. G. Milhano, H. Weigert, Phys. Rev. D 62, 114005 (2000); E. Iancu, A. Leonidov, L. McLerran, hep-ph/0011241; hep-ph/0102009.

[14] E. Levin and K. Tuchin, Nucl. Phys. B573, 833 (2000).

[15] M. A. Braun, Eur. Phys. J. C 16, 337 (2000).

[16] E. Levin and M. Lublinsky, hep-ph/0104108; M. Lublinsky,E. Gotsman, E. Levin and U. Maor, hep-ph/0102321.

[17] E.A. Kuraev, L.N. Lipatov and V.S. Fadin, Sov. Phys. JETP 45, 199 (1977); Ya.Ya. Balitsky and L.N. Lipatov, Sov. J. Nucl. Phys. 28, 22 (1978).

[18] A. A. Belavin, A. M. Polyakov, A. A. Schwartz, and Y. S. Tyupkin, Phys. Lett. B 59, 85 (1975).

[19] G. 't Hooft, Phys. Rev. Lett. 37, 8 (1976); Phys. Rev. D 14, 3432 (1976).

[20] C.G. Callan, R. Dashen, and D.J. Gross, Phys. Lett. B63 334, (1978); Phys. Rev. D 17 2717, (1978).

[21] R. Jackiw and C. Rebbi, Phys. Rev. Lett. 37, 172 (1976).

[22] J. D. Bjorken, Report No. hep-ph/9611421, and references therein.

[23] C.G. Callan, R. Dashen, and D.J. Gross, Phys. Rev. D 19 1826, (1979).

[24] M. A. Shifman, A. I. Vainshtein, V. I. Zakharov, Nucl. Phys. B165, 45 (1980).

[25] I.I. Balitsky and A. V. Yung, Phys. Lett. B 168, 113 (1986); Nucl. Phys. B274, 475 (1986); A. V. Yung, Nucl. Phys. B297, 47 (1988).

[26] I.I. Balitsky and V.M. Braun, Phys. Lett. B314, 237 (1993); Nucl. Phys. B380, 51 (1992); Phys. Rev. D 47, 1879 (1993).

[27] A. Ringwald, F. Schrempp, Phys. Lett. B438, 217 (1998); Nucl. Phys. B507, 134 (1997).

[28] E. Shuryak, I. Zahed, Phys. Rev. D 62, 085014 (2000).

[29] A. Ringwald, Nucl. Phys. B330, 1 (1990); O. Espinosa, Nucl. Phys. B343, 310 (1990); L. McLerran, A. Vainshtein and M. Voloshin, Phys. Rev. D42, 171, 180 (1990); V. A. Rubakov, D. T. Son, and P. G. Tinyakov, Phys. Lett. B287, 342 (1992); A. H. Mueller, Nucl. Phys. B348, 310 (1991); Nucl. Phys. B381,597 (1992); V. I. Zakharov, Nucl. Phys. B383, 218 (1992); B371, 637 (1992); P. Arnold and M. P. Mattis, Phys. Rev. D 44, 3650 (1991); V. V. Khoze and A. Ringwald, Phys. Lett. B 259, 106 (1991); I.I. Balitsky and A. Schafer, Nucl. Phys. B404, 639 (1993).

[30] D. I. Diakonov and M. V. Polyakov, Nucl. Phys. B389, 109 (1993).

[31] D. Kharzeev and E. Levin, Nucl. Phys. B 578, 351 (2000); D. Kharzeev, Yu. Kovchegov and E. Levin, Nucl. Phys. A690, 621 (2001).

[32] M.A. Nowak, E.V. Shuryak, I. Zahed, hep-ph/0012232; E.V. Shuryak, Phys. Lett. B 486, 378 (2000); E.V. Shuryak, I. Zahed, Phys. Rev. D 62, 085014 (2000).

[33] E.V. Shuryak, hep-ph/0101269.

[34] PHOBOs Collaboration, B.B. Back et al., Phys. Rev. Lett. 85, 3100 (2000).

[35] A. Kovner, L. McLerran, and H. Weigert, Phys. Rev. D 52, 6231 (1995); 52, 3809 (1995); M. Gyulassy, L. McLerran, Phys. Rev. C 56, 2219 (1997).

[36] Yu.V. Kovchegov, D. H. Rischke, Phys. Rev. C 56, 1084 (1997).

[37] A. Krasnitz, R. Venugopalan, Report No. hep-ph/0007108; Phys. Rev. Lett. 84, 4309 (2000); Nucl. Phys. B557, 237 (1999).

[38] Yu. V. Kovchegov, hep-ph/0011252.

[39] A.H. Mueller, Nucl. Phys. B307, 34 (1988).

[40] D. J. Gross, R. D. Pisarski, L. G. Yaffe, Rev. Mod. Phys. 53,43 (1981); R.D. Pisarski and L.G. Yaffe, Phys. Lett. B 97, 110 (1980).

[41] R. Baier, A.H. Mueller, D. Schiff, D.T. Son, Phys. Lett. B 502, 51 (2001).

[42] C. Aragão de Carvalho, Nucl. Phys. B183, 182 (1981); E.V. Shuryak, Nucl. Phys. B203, 140 (1982).

[43] J.P. Blaizot and A.H. Mueller, Nucl. Phys. B289, 847 (1987).

[44] L. Frankfurt, V. Guzey and M. Strikman, J. Phys. G 27, R23 (2001) and references therein.

[45] D. Kharzeev and M. Nardi, Phys. Lett. B507, 121 (2001).

[46] A. Ringwald and F. Schrempp, Phys. Lett. B459, 249 (1999).

[47] V.N. Gribov, hep-ph/0006158; Yu.L. Dokshitzer, D. Diakonov and S.I. Troian, Phys. Rept. 58, 269 (1980).

[48] V.A. Novikov, M. A. Shifman, A. I. Vainshtein, and V. I. Zakharov, Usp. Fiz. Nauk 136, 553 (1982).

[49] G. 't Hooft, Phys. Rev. D 14, 3432 (1976); C. W. Bernard, N. H. Christ, A. H. Guth, and E. J. Weinberg, Phys. Rev. D 16, 2967 (1977); L. S. Brown, and D. B. Creamer, Phys. Rev. D 18, 3695 (1978); A. I. Vainshtein, V. I. Zakharov, V. A. Novikov, and M. A. Shifman, Sov. Phys. Usp. 25, 195 (1982).

[50] T. Schäfer, E. V. Shuryak, Rev. Mod. Phys. 70, 323 (1998).

[51] D. I. Diakonov and V. Y. Petrov, Nucl. Phys. B245, 259 (1984).

[52] D. Kharzeev, R.D. Pisarski, M.H.G. Tytgat, Phys. Rev. Lett. 81, 512 (1998); hep-ph/0012012;

D. Kharzeev and R.D. Pisarski, Phys. Rev. D 61, 111901 (2000).

[53] A. H. Mueller, Nucl. Phys. B572, 227 (2000). 
[54] E. Iancu and L. McLerran, hep-ph/0103032.

[55] Yu. V. Kovchegov, E. Levin, L. McLerran, Phys. Rev. C 63, 024903 (2001). 